\title{
The ligase chain reaction as a primary screening tool for the detection of culture positive tuberculosis
}

\author{
T M O'Connor, S Sheehan, B Cryan, N Brennan, C P Bredin
}

\begin{abstract}
Background-The ligase chain reaction Mycobacterium tuberculosis assay uses ligase chain reaction technology to detect tuberculous DNA sequences in clinical specimens. A study was undertaken to determine its sensitivity and specificity as a primary screening tool for the detection of culture positive tuberculosis.

Methods-The study was conducted on 2420 clinical specimens (sputum, bronchoalveolar lavage fluid, pleural fluid, urine) submitted for primary screening for Mycobacterium tuberculosis to a regional medical microbiology laboratory. Specimens were tested in parallel with smear, ligase chain reaction, and culture.

Results-Thirty nine patients had specimens testing positive by the ligase chain reaction assay. Thirty two patients had newly diagnosed tuberculosis, one had a tuberculosis relapse, three had tuberculosis (on antituberculous therapy when tested), and three had healed tuberculosis. In the newly diagnosed group specimens were smear positive in 21 cases $(66 \%)$, ligase chain reaction positive in $\mathbf{3 0}$ cases (94\%), and culture positive in 32 cases $(100 \%)$. Using a positive culture to diagnose active tuberculosis, the ligase chain reaction assay had a sensitivity of $93.9 \%$, a specificity of $99.8 \%$, a positive predictive value of $83.8 \%$, and a negative predictive value of $99.9 \%$.

Conclusions-This study is the largest clinical trial to date to report the efficacy of the ligase chain reaction as a primary screening tool to detect Mycobacterium tuberculosis infection. The authors conclude that ligase chain reaction is a useful primary screening test for tuberculosis, offering speed and discrimination in the early stages of diagnosis and complementing traditional smear and culture techniques.

(Thorax 2000;55:955-957)
\end{abstract}

Keywords: ligase chain reaction; smear; culture; tuberculosis

Correspondence to: Dr T M O'Connor terryoconnor@eircom.net

Received 12 January 2000 Returned to authors 3 May 2000

Revised version received

11 July 2000

Accepted for publication

28 July 2000

Tuberculosis in Ireland causes significant morbidity and mortality with a notification rate of $15 / 100000$ of population reported for $1994{ }^{1}$ One aspect of tuberculosis control that clearly needs improvement is the speed with which the diagnosis is confirmed. If individuals with pul- monary tuberculosis can be identified rapidly, tuberculosis control could potentially be improved by earlier institution of drug treatment, isolation procedures, and contact investigation.

The role of nucleic acid amplification technology in the routine diagnosis of infectious disease is rapidly being established. The use of polymerase chain reaction (PCR) techniques for the detection of Mycobacterium tuberculosis has a reported sensitivity of 55-100\%. ${ }^{2}$ However, contamination of specimens with PCR products can lead to false positive results, and inhibitors of PCR can lead to false negative results. ${ }^{2}$ The ligase chain reaction is an in vitro nucleic acid amplification technique that exponentially amplifies selected DNA sequences. ${ }^{3}$ The Abbott ligase chain reaction assay was first introduced in 1995 for the diagnosis of Chlamydia trachomatis genitourinary infection. ${ }^{4}$ More recently it has been used for the direct detection of $M$ tuberculosis in respiratory specimens. Its specificity and sensitivity have been reported as $74-90 \%$ and $100 \%$, respectively. ${ }^{5-7}$ Chemical inactivation of amplification product minimises the risk of nucleic acid contamination of the specimen and consequent false positive results. However, the ligase chain reaction assay may be oversensitive, as evidenced by positive results in patients with previously treated tuberculosis that is now healed. We have previously reported in a small series its suitability as a primary screening method for testing all samples for the presence of $M$ tuberculosis, ${ }^{8}$ and have since performed assays on all samples sent for $M$ tuberculosis smear and culture testing to a regional medical microbiology laboratory. We undertook this study to evaluate its sensitivity and specificity in culture positive tuberculosis.

\section{Methods}

STUDY SUBJECTS

During the period from 1 April 1997 to 1 June 1998 a total of 2420 clinical specimens (sputum, bronchoalveolar lavage fluid, pleural fluid, urine) were submitted for tuberculosis investigations to the regional medical microbiology laboratory, Cork University Hospital. Specimens were tested in parallel by smear, ligase chain reaction, and culture. The catchment area was the south-west region of the Republic of Ireland (Counties Cork and Kerry) with a population of 546000 .

The microscopy procedure consisted of initial auramine stain for screening, with positive samples being confirmed by Ziehl-Neelsen 
Table 1 Positive tests according to clinical diagnosis $(n=39)$ from April 1997 to fune 1998

\begin{tabular}{|c|c|c|c|}
\hline & Smear positive & Culture positive & $\begin{array}{l}\text { Ligase chain reaction } \\
\text { positive }\end{array}$ \\
\hline \multicolumn{4}{|l|}{ New TB $(n=32)$} \\
\hline Total & $21(66 \%)$ & $32(100 \%)$ & $30(94 \%)$ \\
\hline Sputum $(n=20)$ & $17(85 \%)$ & $20(100 \%)$ & $19(95 \%)$ \\
\hline BAL fluid $(n=7)$ & $3(43 \%)$ & $7(100 \%)$ & $7(100 \%)$ \\
\hline Pleural fluid $(n=3)$ & $0(0 \%)$ & $3(100 \%)$ & $2(67 \%)$ \\
\hline Urine $(n=2)$ & $1(50 \%)$ & $2(100 \%)$ & $2(100 \%)$ \\
\hline \multicolumn{4}{|l|}{ Relapse TB } \\
\hline Sputum $(n=1)$ & $1(100 \%)$ & $1(100 \%)$ & $1(100 \%)$ \\
\hline \multicolumn{4}{|l|}{$\mathrm{TB}$ (on treatment) } \\
\hline Sputum $(n=3)$ & $0(0 \%)$ & $0(0 \%)$ & $3(100 \%)$ \\
\hline \multicolumn{4}{|l|}{ Healed TB } \\
\hline Sputum $(n=3)$ & $0(0 \%)$ & $0(0 \%)$ & $3(100 \%)$ \\
\hline
\end{tabular}

The distribution of specimen type in the group with newly diagnosed tuberculosis is shown. All patients in the other groups had pulmonary tuberculosis. BAL = bronchoalveolar lavage.

stain. The ligase chain reaction based assay for the detection of $M$ tuberculosis complex was run according to the manufacturer's instructions. This uses the nucleic acid amplification method ligase chain reaction to detect the presence of $M$ tuberculosis DNA directly in clinical specimens. Sample preparation involves cell lysis to release DNA. Four oligonucleotide probes in the ligase chain reaction assay recognise and hybridise to a specific target sequence within the chromosomal DNA of the $M$ tuberculosis complex. This nucleic acid sequence encodes for protein antigen $b,{ }^{9}$ a gene sequence which appears to be specific to the $M$ tuberculosis complex and which has been detected in all $M$ tuberculosis complex strains examined to date. ${ }^{10}$ The oligonucleotides are designed to be complementary to the target sequence so that, in the presence of target, the probes will bind adjacent to one another. They can then be enzymatically joined to form the amplification product which subsequently serves as an additional target sequence during further rounds of amplification. Microparticle enzyme immunoassay (MEIA) detects the product of the ligase chain reaction on the Abbott analyser. Culture was performed with Lowenstein-Jensen and pyruvate slopes incubated for 8 and 12 weeks for smear negative and smear positive samples, respectively.

The clinical records of all patients with specimens which were positive on smear, ligase chain reaction, or culture assays for $M$ tuberculosis during a 14 month period (April 1997 to June 1998) were reviewed and their clinical details recorded. Patients were described as having newly diagnosed tuberculosis, relapse of tuberculosis, recently diagnosed tuberculosis (on antituberculous treatment at the time of analysis), or old (healed) tuberculosis as determined by their clinicians. Therapeutic decisions were not made on the basis of ligase chain reaction results. In the case of multiple specimens taken from one patient simultaneously, the various tests were considered positive if at least one of the specimens was positive. However, there were no discrepancies noted between multiple specimens in terms of their smear, ligase chain reaction, or culture results.

STATISTICAL ANALYSIS

The results were calculated using the Graphpad Instat statistical software package (San
Diego, CA, USA) to determine the sensitivity, specificity, and positive and negative predictive values, with their $95 \%$ confidence intervals, for smear and ligase chain reaction assay. The results were compared with the final clinical diagnosis using a positive culture as the gold standard to diagnose active tuberculosis. Positive ligase chain reaction results that were culture negative in patients with tuberculosis on treatment or in patients with old healed tuberculosis were felt to reflect the high sensitivity of the ligase chain reaction assay in detecting non-viable mycobacterial DNA, but were considered to be false positive in the final analysis.

\section{Results}

A total of 2420 specimens were tested in parallel by smear, ligase chain reaction, and culture assays over a 14 month period. Thirty nine patients had specimens which tested positive for smear $(n=22)$, ligase chain reaction $(n=37)$, or culture for $M$ tuberculosis ( $\mathrm{n}=33$; table 1 ). During this period there were two atypical mycobacteria cultured in the laboratory from sputum specimens ( $M$ malmoense and $M$ chelo$n a e)$. Both were smear and ligase chain reaction negative and were considered non-pathogenic.

In the group with newly diagnosed culture positive tuberculosis $(n=32)$ there were 20 men $(63 \%)$ and 12 women $(37 \%)$ with a median age at diagnosis of 43 years (range 18-87). Nineteen of 28 patients on whom smoking information was available $(68 \%)$ were current smokers; 29 patients had pulmonary tuberculosis $(91 \%)$, two had renal tuberculosis (6\%), and one had both pulmonary and renal tuberculosis $(3 \%)$. Specimens were smear positive in 21 cases $(66 \%)$, ligase chain reaction positive in 30 cases $(94 \%)$, and culture positive in 32 cases $(100 \%)$. In the two specimens which were ligase chain reaction negative but culture positive (one sputum, one pleural fluid) there were no recognisable similarities that might suggest a reason for the negative ligase chain reaction assay. In the smear positive group all specimens were ligase chain reaction and culture positive. In the smear negative group $18 \%$ of specimens were ligase chain reaction negative. Discrepant results between ligase chain reaction and culture were in the smear negative group.

In the patient with a relapse of tuberculosis sputum smear, ligase chain reaction, and culture were all positive. In the group with tuberculosis who were receiving treatment before ligase chain reaction testing $(n=3)$ all specimens were sputum and were ligase chain reaction positive but smear and culture negative. Similarly, in the group with old healed tuberculosis $(n=3)$ all specimens were sputum and were ligase chain reaction positive but smear and culture negative (median period elapsed since diagnosis was 2 years (range 2-10)).

In amalgamating the data, using a positive culture as the gold standard for the diagnosis of active tuberculosis, the Abbott ligase chain reaction $M$ tuberculosis assay, when reported as positive, had a sensitivity of $93.9 \%$ ( $95 \%$ confidence interval (CI) 80 to 99 ), a specificity of $99.8 \%$ (95\% CI 99.4 to 99.9 ), a positive 
Table 2 Comparison of smear and ligase chain reaction in culture positive tuberculosis

\begin{tabular}{lll}
\hline & Smear & Ligase chain reaction \\
\hline Sensitivity & $66.7 \%(48.2$ to 82.0$)$ & $93.9 \%(80$ to 99$)$ \\
Specificity & $100 \%(99.8$ to 100$)$ & $99.8 \%(99.4$ to 99.9$)$ \\
Positive predictive value & $100 \%(84.6$ to 100$)$ & $83.8 \%(68.0$ to 93.8$)$ \\
Negative predictive value & $99.5 \%(99.2$ to 99.8$)$ & $99.9 \%(99.7$ to 99.9$)$ \\
p value $\left(\chi^{2}\right.$ test) & $<0.0001$ & $<0.0001$ \\
\hline
\end{tabular}

Values are percentages with $95 \%$ confidence intervals in parentheses.

predictive value of $83.8 \%$ (95\% CI 68.0 to 93.8), and a negative predictive value of $99.9 \%$ ( $95 \%$ CI 99.7 to 99.9 ), table 2 . The $\chi^{2}$ test had a two tailed $\mathrm{p}$ value of $<0.0001$.

\section{Discussion}

The data presented here suggest that the ligase chain reaction assay is a sensitive and specific tool for the detection of culture positive tuberculosis in clinical practice and may, in fact, be more sensitive and specific than other commercially available PCR based diagnostic tests for tuberculosis. ${ }^{511-13}$ However, at a cost of approximately $£ 8.50$ (Irish punts) (equivalent to Euro 10.79) per assay, the cost/benefit ratio of the ligase chain reaction assay remains to be established. Some authors have suggested that clinical diagnosis rather than positive culture results should be used as the reference standard for molecular diagnostic tests in tuberculosis. ${ }^{2}$ However, this study was undertaken specifically to address the clinical efficacy of the ligase chain reaction assay in comparison with culture.

Although a strong presumptive diagnosis of pulmonary tuberculosis can be established by the finding of acid fast bacilli on microscopic examination in $50-60 \%$ of patients with this disease, identification of $M$ tuberculosis in culture is required for confirmation. ${ }^{14}{ }^{15}$ Using conventional solid media for culture, results are not available for 3-6 weeks. Conventionally, the smear positive patient is started on antituberculous treatment immediately, but delay in initiating drug treatment in the smear negative patient may result in progression of the disease.

Although licensed only for respiratory specimens (sputum and bronchoalveolar lavage fluid), the ligase chain reaction assay is sensitive and specific in the diagnosis of extrapulmonary tuberculosis. ${ }^{16}$ The assay was positive in two of three culture positive pleural fluid specimens and in both of two culture positive urine specimens in this trial.

Mycobacterial DNA may remain in the body many years after primary or post-primary infection, so the ligase chain reaction assay may be positive in clinical specimens from a patient with old (healed) tuberculosis and cannot determine therapeutic success or failure following antimicrobial treatment. As with any diagnostic test, results should be interpreted in the context of clinical findings. Its main advantage is speed, with results available within an eight hour working day. Although smear results are usually available within a similar period, the sensitivity of the ligase chain reaction assay is more comparable to that of culture than smear (table 2). In clinical practice this suggests a definite role for the ligase chain reaction assay in patients with negative smear but positive ligase chain reaction results in whom active $M$ tuberculosis infection is clinically suspected. A useful role is also suggested in patients who have smear positive, ligase chain reaction negative specimens. Such results would suggest infection with mycobacteria other than tuberculosis (MOTT) as ligase chain reaction is specific for the $M$ tuberculosis complex. ${ }^{17}$ There were no cases of $M$ bovis in this series. This is consistent with the low prevalence of $M$ bovis infection in humans in this region. ${ }^{18} 19$

As two patients were ligase chain reaction negative and culture positive for $M$ tuberculosis and six were ligase chain reaction positive but smear and culture negative (table 1), it is not anticipated that the ligase chain reaction assay will replace culture as a diagnostic tool for tuberculosis. Rather, the authors conclude that it is a useful primary screening test for tuberculosis, offering speed ( 8 hours vs 3-6 weeks) and discrimination ( $M$ tuberculosis vs MOTT) in the early stages of diagnosis, and complementing traditional smear and culture techniques.

1 Report of the Working Party on Tuberculosis. Dublin: Department of Health, Government of Ireland, 1996: 1.

2 Chin DP, Yajko DM, Hadley WK, et al. Clinical utility of a commercial test based on the polymerase chain reaction for detecting Mycobacterium tuberculosis in respiratory specimens. Am ₹ Respir Crit Care Med 1995;151:1872-7.

3 Lee HH. Ligase chain reaction. Biologicals 1996;24:197-9.

4 Lee HH, Chernesky M, Schachter J, et al. Diagnosis of Chlamydia trachomatis genitourinary infection in women by ligase chain reaction assay of urine. Lancet 1995;345:213-5.

5 Ausina V, Gamboa F, Gazapo E, et al. Evaluation of the semiautomated Abbott LCx Mycobacterium tuberculosis assay for direct detection of Mycobacterium tuberculosis in respiratory specimens. F Clin Microbiol 1997;35:19962002.

6 Marczac J, Pirozynski M, Blachnio A, et al. Application of the ligase chain reaction based test in a prospective detection of Mycobacterium tuberculosis complex. Eur Respir $\mathcal{F}$ (Suppl) 1998;12:222-3S
(1)

7 Garrino MG, Glupczynski Y, Degraux J, et al. Evaluation of the Abbott LCx Mycobacterium tuberculosis assay for direct detection of Mycobacterium tuberculosis complex in human samples. F Clin Microbiol 1999;37:229-32.

8 Sheehan S, Lancaster A, Schroder G, et al. The new Abbott LCx MTB assay used for routine diagnosis of Mycobacterium tuberculosis complex. Ir F Med Sci 1999;168(Suppl):58.

9 Anderson AB, Hansen EB. Structure and mapping of antigenic domains of protein antigen b, a 38,000-molecular weight protein of Mycobacterium tuberculosis. Infect Immun 1989;57:2481-8.

10 Sjobring V, Mecklenberg M, Andersen AB, et al. Polymerase chain reaction for detection of Mycobacterium tuberculosis. $\mathcal{F}$ Clin Microbiol 1990;28:2200-4.

11 Piersimioni C, Callegaro A, Nista D, et al. Comparative evaluation of two commercial amplification assays for direct detection of Mycobacterium tuberculosis complex in respiratory specimens. F Clin Microbiol 1997;35:193-6.

12 Ginesu F, Pirina P, Sechi LA, et al. Microbiological diagnosis of tuberculosis: a comparison of old and new methods. $\mathcal{F}$ Chemother 1998;10:295-300
is of tuberculosis: a comparis

13 Shah S, Miller A, Mastellone A, et al. Rapid diagnosis of tuberculosis in various biopsy and body fluid specimens by the AMPICLOR Mycobacterium tuberculosis polymerase chain reaction test. Chest 1998;113:1190-4.

14 Schluger NW, Rom WN. Current approaches to the diagnosis of active pulmonary tuberculosis. Am $\mathcal{F}$ Respir Crit Care Med 1994;149:264-7.

15 Gordin F, Slutkin G. The validity of acid-fast smears in the diagnosis of pulmonary tuberculosis. Arch Pathol Lab Med 1990;114:1025-7.

16 Gamboa F, Dominguez J, Padilla E, et al. Rapid diagnosis of extrapulmonary tuberculosis by ligase chain reaction amplification. f Clin Microbiol 1998;36:1324-9.

17 Viianen $\mathrm{AH}$, Soini H, Marjamaki M, et al. Ligase chain reaction assay is clinically useful in the discrimination of smearpositive tuberculosis from atypical mycobacterioses. Ann positive tuberculosis fro

18 Cotter TP, O'Shaughnessy E, Sheehan S, et al. Human Mycobacterium bovis infection in the south-west of Ireland 1983-1992: a comparison with $M$ tuberculosis. Ir Med F 1996;89:62-3

19 Cotter TP, Sheehan S, Cryan B, et al. Tuberculosis due to Mycobacterium bovis in humans in the south-west region of Ireland: is there a relationship with infection prevalence in cattle? Tuberc Lung Dis 1996;77:545-8. 